\title{
Starlikeness of General Integral Operator for Meromorphic Multivalent Functions
}

\author{
Pranay Goswami ${ }^{1}$ and Serap Bulut ${ }^{2}$ \\ ${ }^{1}$ School of Liberal Studies, Bharat Ratna Dr B.R. Ambedkar University, Delhi 110006, India \\ ${ }^{2}$ Civil Aviation College, Kocaeli University, Arslanbey Campus, 41285 Kocaeli, Turkey
}

Correspondence should be addressed to Serap Bulut; bulutserap@yahoo.com

Received 31 August 2012; Accepted 30 November 2012

Academic Editor: Jacek Dziok

Copyright (C) 2013 P. Goswami and S. Bulut. This is an open access article distributed under the Creative Commons Attribution License, which permits unrestricted use, distribution, and reproduction in any medium, provided the original work is properly cited.

We introduce a new integral operator $\mathscr{F}_{\gamma, \delta}^{p}\left(f_{1}, \ldots, f_{n}\right)$ for meromorphic multivalent functions. The starlikeness condition of this integral operator is determined. Several special cases are also discussed in the form of corollaries.

\section{Introduction}

Let $\Sigma_{p}$ denote the class of all meromorphic functions of the form

$$
f(z)=\frac{1}{z^{p}}+\sum_{k=0}^{\infty} a_{k} z^{k} \quad(p \in \mathbb{N}:=\{1,2, \ldots\}),
$$

which are analytic and $p$-valent in the punctured open unit disk

$$
\mathbb{U}^{*}=\{z \in \mathbb{C}: 0<|z|<1\}=\mathbb{U} \backslash\{0\},
$$

where $\mathbb{U}$ is the open unit disk $\mathbb{U}=\{z \in \mathbb{C}:|z|<1\}$. In particular, we set $\Sigma_{1}=\Sigma$.

A function $f \in \Sigma_{p}$ is said to be meromorphic $p$ valent starlike and belongs to the class $\mathscr{M} \mathcal{S}_{p}^{*}$, if it satisfies the inequality:

$$
-\mathbb{R}\left\{\frac{z f^{\prime}(z)}{f(z)}\right\}>0 .
$$

A function $f \in \Sigma_{p}$ is said to be meromorphic $p$-valent convex and belongs to the class $\mathscr{M} \mathscr{C}_{p}$, if it satisfies the inequality:

$$
-\mathbb{R}\left\{1+\frac{z f^{\prime \prime}(z)}{f^{\prime}(z)}\right\}>0 .
$$

We note that

$$
f \in M \mathscr{C}_{p} \Longleftrightarrow-\frac{z f^{\prime}}{p} \in \mathscr{M} \mathcal{S}_{p}^{*} .
$$

In particular, we set

$$
\mathscr{M} \mathcal{S}_{1}^{*}=\mathscr{M} \mathcal{S}^{*}, \quad M \mathscr{C}_{1}=\mathscr{M} \mathscr{C}
$$

for the classes of meromorphic starlike and convex functions, respectively.

Analogous to the integral operator defined by Frasin [1] on normalized analytic functions, we now define the following general integral operator for the meromorphic $p$ valent functions in the class $\Sigma_{p}$.

Definition 1. Let $n \in \mathbb{N}, \gamma_{j}, \delta_{j} \in \mathbb{R}_{+} \cup\{0\}$ for all $j=1,2, \ldots, n$ and $\gamma=\left(\gamma_{1}, \ldots, \gamma_{n}\right), \delta=\left(\delta_{1}, \ldots, \delta_{n}\right)$. We introduce a new general integral operator

$$
\mathscr{I}_{\gamma, \delta}^{p}\left(f_{1}, \ldots, f_{n}\right): \Sigma_{p}^{n} \longrightarrow \Sigma_{p},
$$

$$
\begin{aligned}
\mathscr{F}_{\gamma, \delta}^{p}\left(f_{1}, \ldots, f_{n}\right)(z)= & \frac{1}{z^{p+1}} \int_{0}^{z} \prod_{j=1}^{n}\left(-\frac{u^{p+1}}{p} f_{j}^{\prime}(u)\right)^{\gamma_{j}} \\
& \times\left(u^{p} f_{j}(u)\right)^{\delta_{j}} d u \quad\left(z \in \mathbb{U}^{*}\right) .
\end{aligned}
$$


Remark 2. The integral operator introduced here generalizes the following integral operators.

(i) If $p=1$, then we have the integral operator

$$
\begin{aligned}
\mathscr{I}_{\gamma, \delta}\left(f_{1}, \ldots, f_{n}\right)(z)= & \frac{1}{z^{2}} \int_{0}^{z} \prod_{j=1}^{n}\left(-u^{2} f_{j}^{\prime}(u)\right)^{\gamma_{j}} \\
& \times\left(u f_{j}(u)\right)^{\delta_{j}} d u
\end{aligned}
$$

introduced and studied by Bulut and Goswami [2].

(ii) If $\delta=(0, \ldots, 0)$, then it reduces to the integral operator

$\mathscr{J}_{\gamma}^{p}\left(f_{1}, \ldots, f_{n}\right)(z)=\frac{1}{z^{p+1}} \int_{0}^{z} \prod_{j=1}^{n}\left(-\frac{u^{p+1}}{p} f_{j}^{\prime}(u)\right)^{\gamma_{j}} d u$.

(iii) If $\gamma=(0, \ldots, 0)$, then it reduces to the integral operator

$$
\mathscr{G}_{\delta}^{p}\left(f_{1}, \ldots, f_{n}\right)(z)=\frac{1}{z^{p+1}} \int_{0}^{z} \prod_{j=1}^{n}\left(u^{p} f_{j}(u)\right)^{\delta_{j}} d u .
$$

(iv) For $n=1, \gamma_{1}=\gamma, \delta_{1}=\delta$, and $f_{1}=f$, it reduces to the new integral operator

$$
\mathscr{I}_{\gamma, \delta}^{p}(f)(z)=\frac{1}{z^{p+1}} \int_{0}^{z}\left(-\frac{u^{p+1}}{p} f^{\prime}(u)\right)^{\gamma}\left(u^{p} f(u)\right)^{\delta} d u .
$$

The integral operators defined in (ii) and (iii) are introduced and studied by Mohammed and Darus [3] (also the case $p=1$ in (ii) and (iii) is defined in [4] and [12], resp.). Furthermore, the case $p=1$ for the integral operator in (iv) is defined by Bulut and Goswami [2]. For the recent developments on meromorphic functions, refer to [5-10].

For the starlikeness of the integral operator $\mathscr{G}_{\gamma, \delta}^{P}\left(f_{1}, \ldots\right.$, $f_{n}$ ) defined in Definition 1, we need to use the following lemma.

Lemma 3 (see $[11])$. Let $\psi: \mathbb{C}^{2} \rightarrow \mathbb{C}$ satisfy the following condition:

$$
\mathbb{R}\{\psi(i s, t)\} \leq 0, \quad\left(s, t \in \mathbb{R} ; t \leq-\frac{|a+i s|^{2}}{2}\right) .
$$

If the function $h(z)=a+h_{1} z+h_{2} z^{2}+\cdots$, where $\mathbb{R}(a)>0$, is analytic in $\mathbb{U}$ and

$$
\mathbb{R}\left\{\psi\left(h(z), z h^{\prime}(z)\right)\right\}>0 \quad(z \in \mathbb{U})
$$

then

$$
\mathbb{R}\{h(z)\}>0 \quad(z \in \mathbb{U}) .
$$

\section{Starlikeness of the Integral}

Operator $\mathscr{I}_{\gamma, \delta}^{p}\left(f_{1}, \ldots, f_{n}\right)$

In this section, we investigate sufficient conditions for the meromorphically starlikeness of the integral operator $\mathscr{I}_{\gamma, \delta}^{p}\left(f_{1}, \ldots, f_{n}\right)$ which is defined in Definition 1.

Theorem 4. Let $f_{j} \in \Sigma_{p}, \gamma_{j}, \delta_{j} \in \mathbb{R}_{+} \cup\{0\}$ for all $j=$ $1,2, \ldots, n$ and $\gamma=\left(\gamma_{1}, \ldots, \gamma_{n}\right), \delta=\left(\delta_{1}, \ldots, \delta_{n}\right)$. If

$$
\mathbb{R}\left\{-\gamma_{j} \frac{z f_{j}^{\prime \prime}(z)}{f_{j}^{\prime}(z)}-\delta_{j} \frac{z f_{j}^{\prime}(z)}{f_{j}(z)}\right\}>-\frac{p}{n}+(p+1) \gamma_{j}+p \delta_{j}
$$

for all $j=1,2, \ldots, n$, then the general integral operator $\mathscr{I}_{\gamma, \delta}^{p}\left(f_{1}, \ldots, f_{n}\right)$ defined in Definition 1 belongs to the meromorphic starlike function class $\mathscr{M} \mathcal{S}_{p}^{*}$.

Proof. For the sake of simplicity, in the proof, we shall write $\mathscr{F}(z)$ instead of $\mathscr{J}_{\gamma, \delta}^{p}\left(f_{1}, \ldots, f_{n}\right)(z)$. From $(7)$, it is easy to see that

$$
\begin{aligned}
z^{p+1} \mathscr{F}^{\prime}(z)+(p+1) z^{p} \mathscr{F}(z) \\
=\prod_{j=1}^{n}\left(-\frac{z^{p+1} f_{j}^{\prime}(z)}{p}\right)^{\gamma_{j}}\left(z^{p} f_{j}(z)\right)^{\delta_{j}} .
\end{aligned}
$$

Differentiating the above equality with respect to $z$, we obtain

$$
\begin{aligned}
& z^{p+1} \mathscr{F}^{\prime \prime}(z)+2(p+1) z^{p \mathscr{F}^{\prime}}(z)+p(p+1) z^{p-1} \mathscr{F}(z) \\
&= \sum_{j=1}^{n}\left\{\gamma_{j}\left(-\frac{z^{p+1} f_{j}^{\prime}(z)}{p}\right)^{\gamma_{j}}\left(z^{p} f_{j}(z)\right)^{\delta_{j}}\right. \\
& \times\left(\frac{p+1}{z}+\frac{f_{j}^{\prime \prime}(z)}{f_{j}^{\prime}(z)}\right)+\delta_{j}\left(-\frac{z^{p+1} f_{j}^{\prime}(z)}{p}\right)^{\gamma_{j}} \\
&\left.\times\left(z^{p} f_{j}(z)\right)^{\delta_{j}}\left(\frac{p}{z}+\frac{f_{j}^{\prime}(z)}{f_{j}(z)}\right)\right\} \\
& \times \prod_{\substack{s=1 \\
(s \neq j)}}^{n}\left(-\frac{z^{p+1} f_{s}^{\prime}(z)}{p}\right)^{\gamma_{s}}\left(z^{p} f_{s}(z)\right)^{\delta_{s}} .
\end{aligned}
$$

From (16) and (17), we get

$$
\begin{aligned}
\frac{z^{p+1} \mathscr{F}^{\prime \prime}(z)+2(p+1) z^{p} \mathscr{F}^{\prime}(z)+p(p+1) z^{p-1} \mathscr{F}(z)}{z^{p+1} \mathscr{F}^{\prime}(z)+(p+1) z^{p \mathscr{F}}(z)} \\
=\sum_{j=1}^{n}\left\{\gamma_{j}\left(\frac{p+1}{z}+\frac{f_{j}^{\prime \prime}(z)}{f_{j}^{\prime}(z)}\right)+\delta_{j}\left(\frac{p}{z}+\frac{f_{j}^{\prime}(z)}{f_{j}(z)}\right)\right\}
\end{aligned}
$$


or equivalently

$$
\begin{aligned}
& \frac{z^{2} \mathscr{F}^{\prime \prime}(z)+2(p+1) z \mathscr{F}^{\prime}(z)+p(p+1) \mathscr{F}(z)}{z \mathscr{F}^{\prime}(z)+(p+1) \mathscr{F}(z)} \\
& =\sum_{j=1}^{n}\left\{\gamma_{j}\left(p+1+\frac{z f_{j}^{\prime \prime}(z)}{f_{j}^{\prime}(z)}\right)+\delta_{j}\left(p+\frac{z f_{j}^{\prime}(z)}{f_{j}(z)}\right)\right\} .
\end{aligned}
$$

After some calculations, we have

$$
\begin{aligned}
&-\frac{z^{2} \mathscr{F}^{\prime \prime}(z)+(p+2) z \mathscr{F}^{\prime}(z)}{z \mathscr{F}^{\prime}(z)+(p+1) \mathscr{F}(z)} \\
&=p+\sum_{j=1}^{n}\left\{\gamma_{j}\left(-p-1-\frac{z f_{j}^{\prime \prime}(z)}{f_{j}^{\prime}(z)}\right)\right. \\
&\left.+\delta_{j}\left(-p-\frac{z f_{j}^{\prime}(z)}{f_{j}(z)}\right)\right\} .
\end{aligned}
$$

We can write the left-hand side of (20) as the following:

$$
\begin{gathered}
-\frac{\left(z \mathscr{F}^{\prime}(z) / \mathscr{F}(z)\right)\left(\left(z \mathscr{F}^{\prime \prime}(z) / \mathscr{F}^{\prime}(z)\right)+p+2\right)}{\left(z \mathscr{F}^{\prime}(z) / \mathscr{F}(z)\right)+p+1} \\
=p+\sum_{j=1}^{n}\left\{\gamma_{j}\left(-p-1-\frac{z f_{j}^{\prime \prime}(z)}{f_{j}^{\prime}(z)}\right)\right. \\
\left.+\delta_{j}\left(-p-\frac{z f_{j}^{\prime}(z)}{f_{j}(z)}\right)\right\} .
\end{gathered}
$$

Now we define a regular function $h(z)$ by

$$
h(z)=-\frac{z \mathscr{F}^{\prime}(z)}{\mathscr{F}(z)},
$$

and $h(0)=p$. Differentiating (22) logarithmically, we obtain

$$
-h(z)+\frac{z h^{\prime}(z)}{h(z)}=1+\frac{z \mathscr{F}^{\prime \prime}(z)}{\mathscr{F}^{\prime}(z)} .
$$

From (21), (22), and (23), we have

$$
\begin{aligned}
& h(z)+\frac{z h^{\prime}(z)}{-h(z)+p+1} \\
&=p+\sum_{j=1}^{n}\left\{\gamma_{j}\left(-p-1-\frac{z f_{j}^{\prime \prime}(z)}{f_{j}^{\prime}(z)}\right)\right. \\
&\left.\quad+\delta_{j}\left(-p-\frac{z f_{j}^{\prime}(z)}{f_{j}(z)}\right)\right\} .
\end{aligned}
$$

Let us put

$$
\psi(u, v)=u+\frac{v}{-u+p+1} .
$$

From (15), (24) and (25), we get

$$
\mathbb{R}\left\{\psi\left(h(z), z h^{\prime}(z)\right)\right\}
$$

$$
\begin{array}{r}
=p+\sum_{j=1}^{n}\left\{\gamma_{j}\left(-p-1-\mathbb{R}\left\{\frac{z f_{j}^{\prime \prime}(z)}{f_{j}^{\prime}(z)}\right\}\right)\right. \\
\left.+\delta_{j}\left(-p-\mathbb{R}\left\{\frac{z f_{j}^{\prime}(z)}{f_{j}(z)}\right\}\right)\right\}
\end{array}
$$

$$
=p-\sum_{j=1}^{n}\left((p+1) \gamma_{j}+p \delta_{j}\right)
$$

$$
+\sum_{j=1}^{n} \mathbb{R}\left\{-\gamma_{j} \frac{z f_{j}^{\prime \prime}(z)}{f_{j}^{\prime}(z)}-\delta_{j} \frac{z f_{j}^{\prime}(z)}{f_{j}(z)}\right\}
$$

$$
\begin{aligned}
> & p-\sum_{j=1}^{n}\left((p+1) \gamma_{j}+p \delta_{j}\right) \\
& +\sum_{j=1}^{n}\left(-\frac{p}{n}+(p+1) \gamma_{j}+p \delta_{j}\right)=0 .
\end{aligned}
$$

Now we have to show that

$$
\mathbb{R}\{\psi(i s, t)\} \leq 0, \quad\left(s, t \in \mathbb{R} ; t \leq-\frac{p^{2}+s^{2}}{2}\right) .
$$

From (25), we have

$$
\begin{aligned}
\mathbb{R}\{\psi(i s, t)\} & =\mathbb{R}\left\{i s+\frac{t}{-i s+p+1}\right\} \\
& =\frac{t(p+1)}{s^{2}+(p+1)^{2}} \\
& \leq-\frac{\left(p^{2}+s^{2}\right)(p+1)}{2\left(s^{2}+(p+1)^{2}\right)}<0 .
\end{aligned}
$$

Thus using Lemma 3, and from (26) and (28), we conclude that $\mathbb{R}\{h(z)\}>0$, and so

$$
-\mathbb{R}\left\{\frac{z \mathscr{F}^{\prime}(z)}{\mathscr{F}(z)}\right\}>0,
$$

that is, the integral operator $\mathscr{F}=\mathscr{I}_{\gamma, \delta}^{p}\left(f_{1}, \ldots, f_{n}\right)$ is a meromorphic multivalent starlike function.

Putting $p=1$ in Theorem 4, we get the following Corollary. 
Corollary 5 (see [2, Theorem 2.1]). Let $f_{j} \in \Sigma, \gamma_{j}, \delta_{j} \in \mathbb{R}_{+} \cup$ $\{0\}$ for all $j=1,2, \ldots, n$ and $\gamma=\left(\gamma_{1}, \ldots, \gamma_{n}\right), \delta=\left(\delta_{1}, \ldots, \delta_{n}\right)$. If

$$
\mathbb{R}\left\{-\gamma_{j} \frac{z f_{j}^{\prime \prime}(z)}{f_{j}^{\prime}(z)}-\delta_{j} \frac{z f_{j}^{\prime}(z)}{f_{j}(z)}\right\}>-\frac{1}{n}+2 \gamma_{j}+\delta_{j}
$$

for all $j=1,2, \ldots, n$, then the general integral operator $\mathscr{I}_{\gamma, \delta}\left(f_{1}, \ldots, f_{n}\right)$ defined in (8) belongs to the meromorphically starlike function class $\mathscr{M} \mathcal{S}^{*}$.

Taking $\delta=(0, \ldots, 0)$ in Theorem 4 , we get the following.

Corollary 6. Let $f_{j} \in \Sigma_{p}, \gamma_{j}>0$ for all $j=1,2, \ldots, n$ and $\gamma=\left(\gamma_{1}, \ldots, \gamma_{n}\right)$. If

$$
\mathbb{R}\left\{-\gamma_{j} \frac{z f_{j}^{\prime \prime}(z)}{f_{j}^{\prime}(z)}\right\}>-\frac{p}{n}+(p+1) \gamma_{j}
$$

for all $j=1,2, \ldots, n$, then the general integral operator $\mathcal{J}_{\gamma}^{p}\left(f_{1}, \ldots, f_{n}\right)$ defined in (9) belongs to the meromorphically starlike function class $\mathscr{M} \mathcal{S}_{p}^{*}$.

Remark 7. If we set $p=1$ in Corollary 6 , then we have [12, Theorem 2.1].

Next, putting $\gamma=(0, \ldots, 0)$ in Theorem 4 , we get the following.

Corollary 8. Let $f_{j} \in \Sigma_{p}, \delta_{j}>0$ for all $j=1,2, \ldots, n$ and $\delta=\left(\delta_{1}, \ldots, \delta_{n}\right)$. If

$$
\mathbb{R}\left\{-\delta_{j} \frac{z f_{j}^{\prime}(z)}{f_{j}(z)}\right\}>-\frac{p}{n}+p \delta_{j}
$$

for all $j=1,2, \ldots, n$, then the general integral operator $\mathscr{G}_{\delta}^{p}\left(f_{1}, \ldots, f_{n}\right)$ defined in (10) belongs to the meromorphically starlike function class $\mathscr{M} \mathcal{S}_{p}^{*}$.

Further taking $n=1, \gamma_{1}=\gamma, \delta_{1}=\delta$, and $f_{1}=f$ in Theorem 4, we have the following.

Corollary 9. Let $f \in \Sigma_{p}$ and $\gamma, \delta>0$. If

$$
\mathbb{R}\left\{-\gamma \frac{z f^{\prime \prime}(z)}{f^{\prime}(z)}-\delta \frac{z f^{\prime}(z)}{f(z)}\right\}>-p+(p+1) \gamma+p \delta
$$

then the general integral operator $\mathscr{I}_{\gamma, \delta}^{p}(f)$ defined in (11) belongs to the meromorphically starlike function class $\mathscr{M} \mathcal{S}_{p}^{*}$.

\section{References}

[1] B. A. Frasin, "Order of convexity and univalency of general integral operator," Journal of the Franklin Institute, vol. 348, no. 6, pp. 1013-1019, 2011.
[2] S. Bulut and P. Goswami, "Starlikeness properties of general integral operator for meromorphic univalent functions," Southeast Asian Bulletin of Mathematics, vol. 37, 2013.

[3] A. Mohammed and M. Darus, "The order of starlikeness of new p-valent meromorphic functions," International Journal of Mathematical Analysis, vol. 6, no. 25-28, pp. 1329-1340, 2012.

[4] A. Mohammed and M. Darus, "A new integral operator for meromorphic functions," Acta Universitatis Apulensis. Mathematics. Informatics, no. 24, pp. 231-238, 2010.

[5] A. Mohammed and M. Darus, "New criteria for meromorphic functions," International Journal of Applied Mathematics \& Statistics, vol. 33, no. 3, 2013.

[6] Y. Sun, W.-P. Kuang, and Z.-G. Wang, "On meromorphic starlike functions of reciprocal order $\alpha$," Bulletin of the Malaysian Mathematical Sciences Society, vol. 35, no. 2, pp. 469-477, 2012.

[7] Z.-G. Wang, Z.-H. Liu, and A. Catas, "On neighborhoods and partial sums of certain meromorphic multivalent functions," Applied Mathematics Letters, vol. 24, no. 6, pp. 864-868, 2011.

[8] Z.-G. Wang, Z.-H. Liu, and R.-G. Xiang, "Some criteria for meromorphic multivalent starlike functions," Applied Mathematics and Computation, vol. 218, no. 3, pp. 1107-1111, 2011.

[9] Z.-G. Wang, Y. Sun, and N. Xu, "Some properties of certain meromorphic close-to-convex functions," Applied Mathematics Letters, vol. 25, no. 3, pp. 454-460, 2012.

[10] Z.-G. Wang, Y. Sun, and Z.-H. Zhang, "Certain classes of meromorphic multivalent functions," Computers and Mathematics with Applications, vol. 58, no. 7, pp. 1408-1417, 2009.

[11] S. S. Miller and P. T. Mocanu, Differential Subordinations: Theory and Applications, vol. 225 of Monographs and Textbooks in Pure and Applied Mathematics, Marcel Dekker, New York, NY, USA, 2000.

[12] A. Mohammed and M. Darus, "Starlikeness properties of a new integral operator for meromorphic functions," Journal of Applied Mathematics, vol. 2011, Article ID 804150, 8 pages, 2011. 


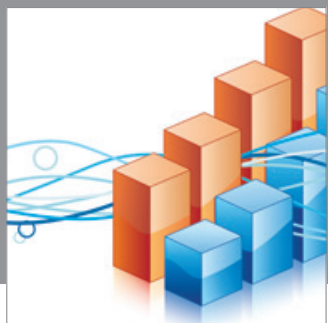

Advances in

Operations Research

mansans

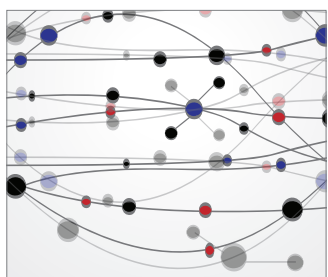

The Scientific World Journal
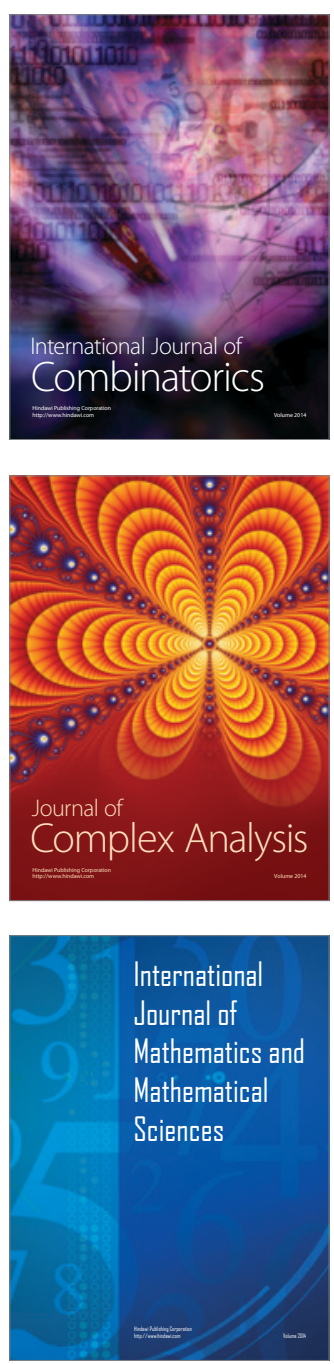
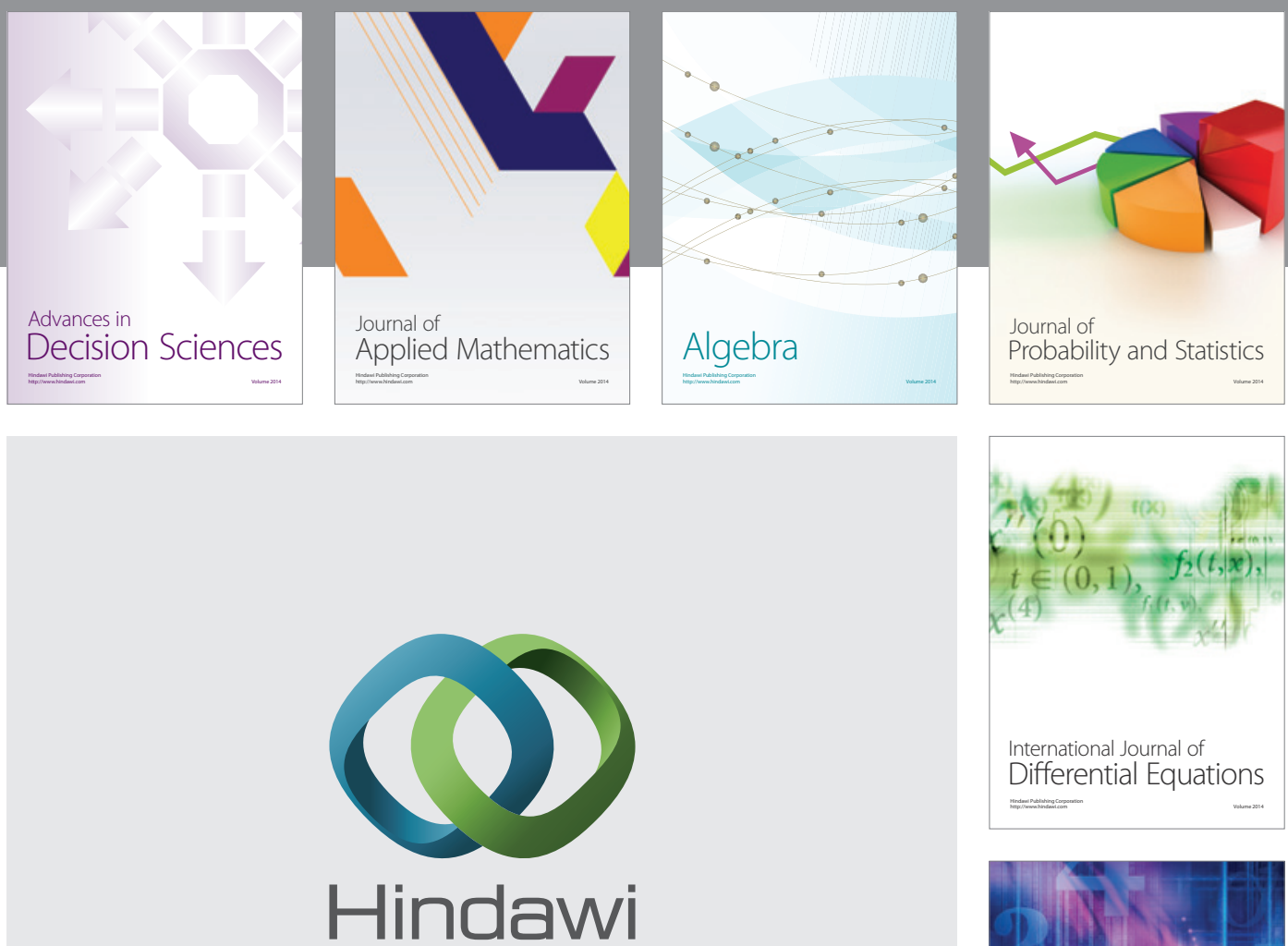

Submit your manuscripts at http://www.hindawi.com
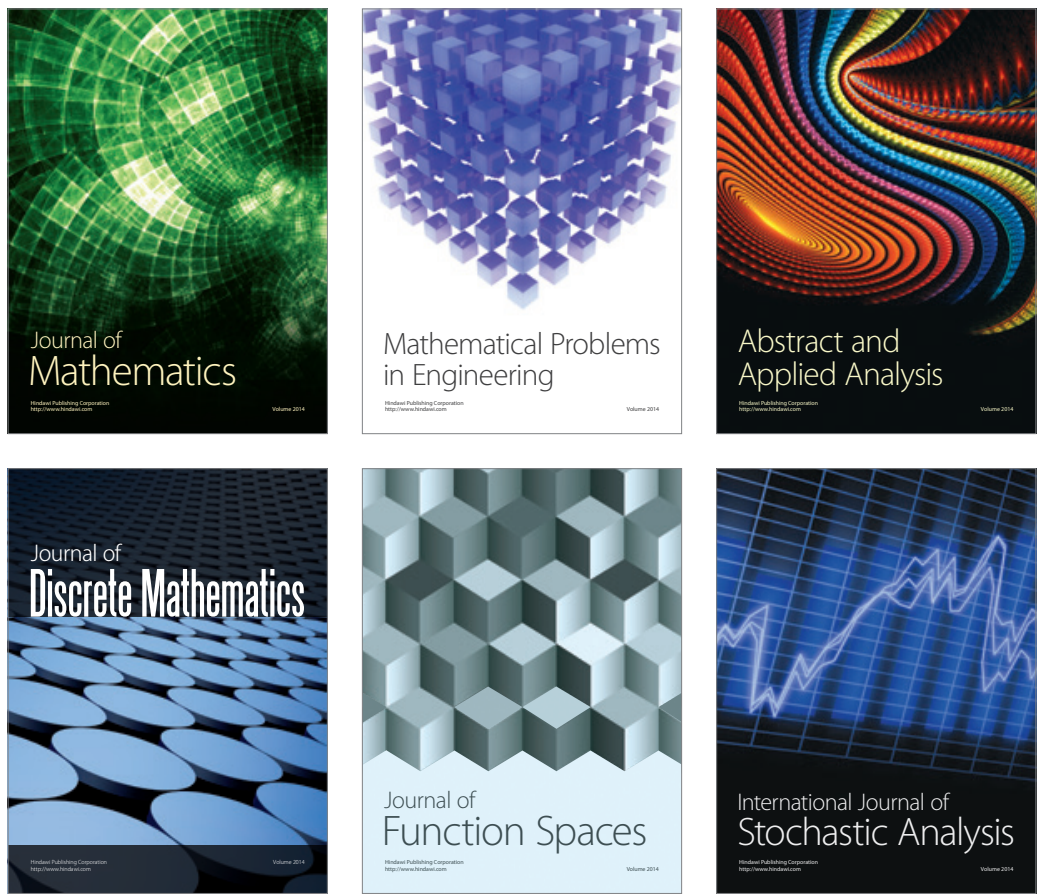

Journal of

Function Spaces

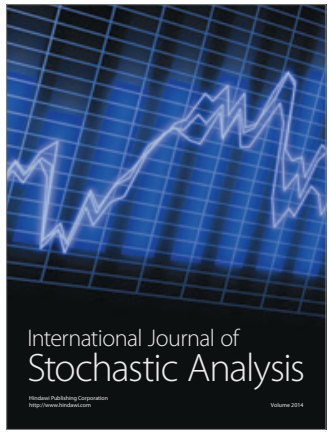

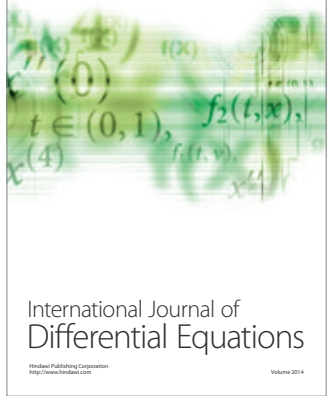
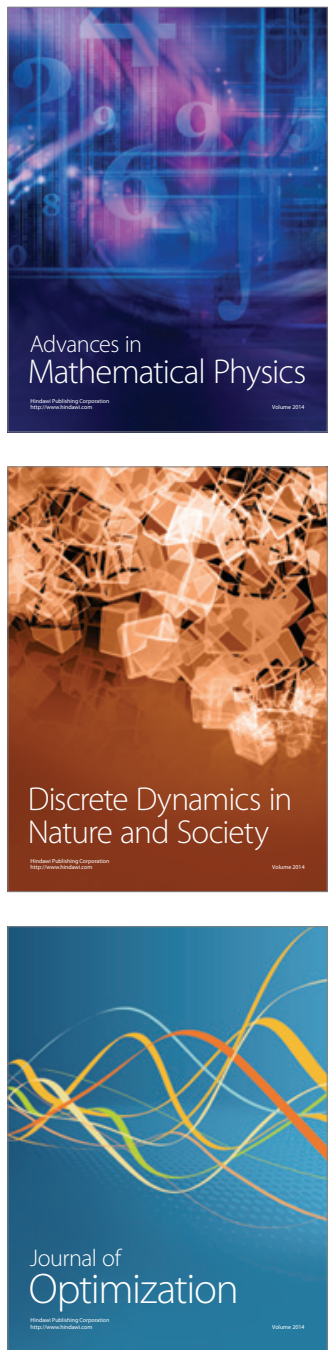